\title{
Unique Challenges Testing SDRs for Space
}

\author{
David Chelmins \\ NASA Glenn \\ Research Center \\ MS 54-4, 21000 \\ Brookpark Road \\ Cleveland, OH 44135 \\ 216-433-3304 \\ dchelmins@nasa.gov
}

\author{
Joseph Downey \\ NASA Glenn Research \\ Center \\ MS 54-4, 21000 Brookpark \\ Road \\ Cleveland, OH 44135 \\ 216-433-6067 \\ joseph.a.downey@nasa.gov
}

\author{
Sandra K. Johnson \\ NASA Glenn Research \\ Center \\ MS 54-1, 21000 Brookpark \\ Road \\ Cleveland, OH 44135 \\ 216-433-8016 \\ sandra.k.johnson@nasa.gov
}

\author{
Jennifer Nappier \\ NASA Glenn Research \\ Center \\ MS 54-1, 21000 Brookpark \\ Road \\ Cleveland, OH 44135 \\ 216-433-6521 \\ jennifer.m.nappier@nasa.gov
}

\begin{abstract}
This paper describes the approach used by the Space Communication and Navigation (SCaN) Testbed team to qualify three Software Defined Radios (SDR) for operation in space and the characterization of the platform to enable upgrades on-orbit. The three SDRs represent a significant portion of the new technologies being studied on board the SCAN Testbed, which is operating on an external truss on the International Space Station (ISS). The SCaN Testbed provides experimenters an opportunity to develop and demonstrate experimental waveforms and applications for communication, networking, and navigation concepts and advance the understanding of developing and operating SDRs in space.
\end{abstract}

Qualifying a Software Defined Radio for the space environment requires additional consideration versus a hardware radio. Tests that incorporate characterization of the platform to provide information necessary for future waveforms, which might exercise extended capabilities of the hardware, are needed. The development life cycle for the radio follows the software development life cycle, where changes can be incorporated at various stages of development and test. It also enables flexibility to be added with minor additional effort. Although this provides tremendous advantages, managing the complexity inherent in a software implementation requires a testing beyond the traditional hardware radio test plan.

Due to schedule and resource limitations and parallel development activities, the subsystem testing of the SDRs at the vendor sites was primarily limited to typical fixed transceiver type of testing. NASA's Glenn Research Center (GRC) was responsible for the integration and testing of the SDRs into the SCaN Testbed system and conducting the investigation of the SDR to advance the technology to be accepted by missions. This paper will describe the unique tests that were conducted at both the subsystem and system level, including environmental testing, and present results. For example, test waveforms were developed to measure the gain of the transmit system across the tunable frequency band. These were used during thermal vacuum testing to enable characterization of the integrated system in the wide operational temperature range of space. Receive power indicators were used for Electromagnetic Interference tests (EMI) to understand the platform's susceptibility to external interferers independent of the waveform. Additional approaches and lessons learned during the SCaN Testbed subsystem and system level testing will be discussed that may help future SDR integrators.
TABle OF CONTENTS

1. INTRODUCTION 1

2. SCAN TESTBED SYSTEM OVERVIEW ...............1

3. GENERAL TEST APPROACH...............................3

4. GENERAL DYNAMICS SDR TEST SUMMARY ...4

5. JPL SDR TEST SUMMARY ................................5

5. HARRIS SDR TEST SUMMARY ..........................7

6. SUMMARY AND CONCLUSION...........................8

7. FUTURE WORK ..............................................8

REFERENCES....................................................8

\section{INTRODUCTION}

As part of the subsystem and system level verification and test of the three SDRs for the SCaN Testbed system, knowledge was gained on improving the approach for testing software defined platforms and waveforms that is distinct from typical hardware radio tests. This paper captures, at a high level, some of this knowledge for future SDR platform and waveform developers. It also provides examples of software and firmware modifications implemented during the test campaign that enabled improved performance.

This paper is comprised of a brief overview of the SCaN Testbed system and a description of each SDR in Section 2. Sections 3-5 describe the unique test approach for each SDR, describe software updates, and provide lessons learned and suggestions for future SDR test programs. Section 6 provides a summary of the paper and conclusions. Section 7 captures Future Work envisioned to perform onorbit testing and compare results with ground test data.

\section{SCAN TESTBED SYSTEM OVERVIEW}

The objective of the SCAN Testbed is to study the development, testing, and operation of Software Defined Radios and their associated applications in the operational space environment to reduce cost and risk for future space missions. NASA is soliciting universities, industry, and other Government agencies to propose experiments using the testbed via a Cooperative Agreement Notice and Announcement of Opportunity [1]. SDRs offer the promise 
of in-flight reconfigurability, autonomy, and an evolution to cognitive operation. The SCaN Testbed flight system, operating on an external truss on the ISS, contains three SDRs capable of operating at S-, Ka- and L-band, along with the accompanying RF/antenna systems. The SCaN Testbed system also consists of a Control Center located at NASA's Glenn Research Center in Cleveland, Ohio. The path for commanding the SCaN Testbed flight system and receiving Health and Status $(H \& S)$ telemetry from the flight system is through the ISS command/telemetry system, and is referred to as the "Primary Data Path". The data path using the SCaN Testbed RF system, which operates directly with the NASA's Tracking and Data Relay Satellite System (TDRSS) [2], is referred to as the "Experiment Data Path". See Figure 1. Having a separate path to command the SDRs and receive telemetry from the RF path is necessary as a payload on ISS, but it provides an additional benefit for loading and testing new waveforms on the SDRs by enabling a redundant file transfer path if new waveform updates encounter unexpected behavior. transmit information via radio frequency $(\mathrm{RF})$ signals. Table 1 provides a summary of the SDR platform characteristics.

General Dynamics (GD) Corporation SDR - The General Dynamics SDR was developed under a competed cooperative agreement with NASA, where GD funded a significant portion of the development costs. This approach is ideal for technology development efforts such as the SCaN Testbed, where the developer shares the risk of the new development, while also benefiting from NASA's investment. The GD SDR is capable of full-duplex, TDRSScompatible, STRS-compliant S-band communications. The GD SDR leverages developments of the TDRSS fourthgenerational transponder.

Jet Propulsion Laboratory (JPL) SDR - The JPL SDR was procured using an existing development task contract between NASA and JPL. It leverages the development of the Electra [4] radio. It is capable of full-duplex, TDRSScompatible, STRS-compliant S-band communications and

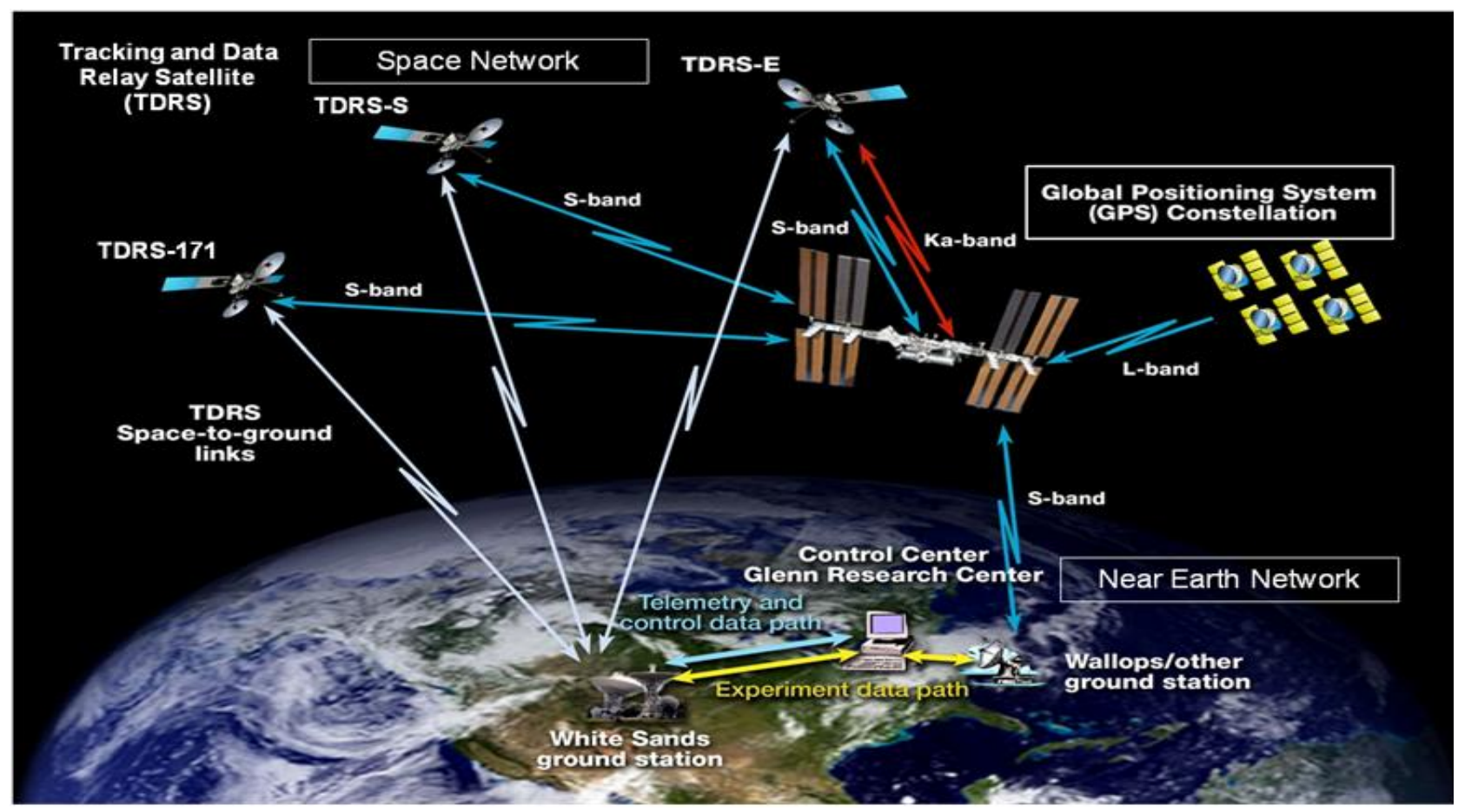

Figure 1. SCaN Testbed System Architecture

At the core of the Flight System are three unique software defined radios provided by government and industry partners - Figure 2. Each of the three SDRs has an Operating Environment (OE), which includes an operating system and infrastructure services to applications and waveforms in accordance with the Space Telecommunications Radio System Standard (STRS). The OE middleware (compliant with the STRS Architecture Standard [3]) abstracts the SDR hardware from the waveform application software. In addition to the OE, each SDR runs STRS-compliant waveform applications, which implement the unique capabilities of the radio to receive and receive-only GPS L-band for navigation. The S-band and GPS can be operated simultaneously. [5]

Harris Corporation SDR - The Harris SDR was also developed under a competed Cooperative Agreement with NASA. It is capable of full-duplex TDRSS-compatible, STRS-compliant Ka-band communications. The Harris SDR is NASA's first TDRSS-compatible flight Ka-band transmitter/receiver system. Harris Corporation managed to design, build, unit test, and deliver this SDR in only 14 months. [6] 
SDR

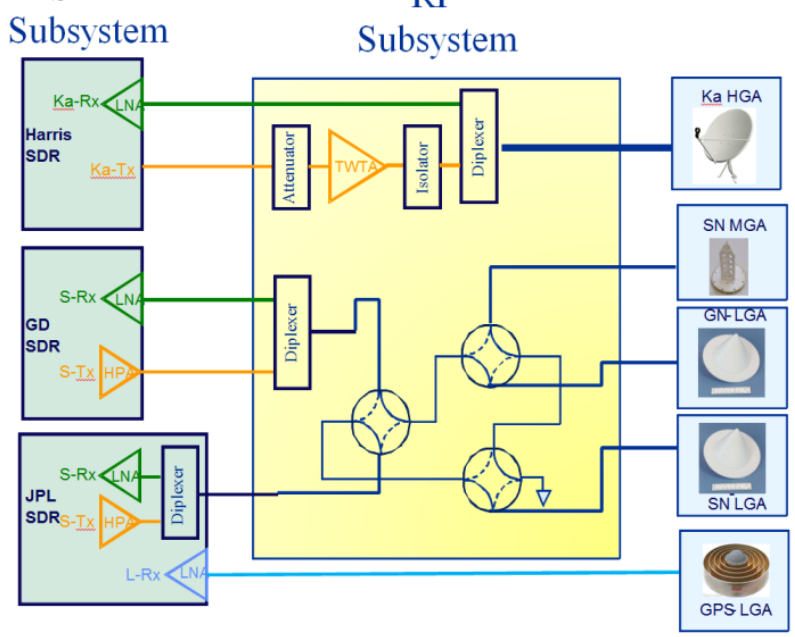

Figure 2. SCaN Testbed Flight System Diagram

Table 1 - SDR Characteristics Comparison

\begin{tabular}{|c|c|c|c|}
\hline $\begin{array}{l}\text { Industry } \\
\text { Partner }\end{array}$ & GD & $\begin{array}{c}\text { JPL \& } \\
\text { L-3/ } \\
\text { Cincinnati } \\
\text { Electronics }\end{array}$ & Harris \\
\hline Freq Band & S-band & $\begin{array}{l}\text { S-band and } \\
\text { L-band }\end{array}$ & Ka-band \\
\hline $\begin{array}{l}\text { WF FPGA } \\
\text { Capacity }\end{array}$ & $\begin{array}{l}\text { Virtex II } \\
\text { 3M gates }\end{array}$ & $\begin{array}{c}\text { Virtex II } \\
2 \text { x } 3 \mathrm{M} \text { gates }\end{array}$ & $\begin{array}{c}\text { Virtex IV } \\
4 \mathrm{x} 6 \mathrm{M} \text { gates }\end{array}$ \\
\hline Processor & Coldfire & SPARC & $\begin{array}{l}\text { AITech, } \\
\text { PPC }\end{array}$ \\
\hline os & VxWorks & RTEMS & VxWorks \\
\hline $\begin{array}{l}\text { NV memory } \\
\text { MByte }\end{array}$ & 4 & $\begin{array}{c}3 \times 512 \\
\text { flash }\end{array}$ & $\begin{array}{l}1 \text { boot } \\
64 \text { user }\end{array}$ \\
\hline $\begin{array}{c}\text { RAM } \\
\text { MByte }\end{array}$ & 128 & $3 \times 128$ & 128 \\
\hline $\begin{array}{c}\mathrm{C \& T} \\
\text { Interface }\end{array}$ & 1553 & 1553 & SpaceWire \\
\hline $\begin{array}{c}\text { Data } \\
\text { Interface }\end{array}$ & SpaceWire & SpaceWire & SpaceWire \\
\hline $\begin{array}{c}\text { Abstraction } \\
\text { Layer }\end{array}$ & $\begin{array}{c}\text { STRS } \\
\text { Compliant }\end{array}$ & $\begin{array}{c}\text { STRS } \\
\text { Compliant }\end{array}$ & $\begin{array}{c}\text { STRS } \\
\text { Compliant }\end{array}$ \\
\hline
\end{tabular}

\section{General TeSt ApProach}

\section{Standard Space Radio Test Approach}

When an SDR is tested with an integrated, functional waveform, the test approach is very similar to the test approach for a hardware-based transceiver. In the case of the SCaN Testbed SDRs, all SDRs had a suite of TDRSScompatible waveforms developed prior to launch and used for the subsystem (SDR alone) and system level tests. [7] Typical tests include BER characterization, receiver acquisition thresholds and timing, transmitter error vector magnitudes, transmit power level, and transmit spectrum plots. Many of these tests are also completed during thermal vacuum testing, in which the SDRs are placed in a vacuum tank and the temperature was driven to the hot and cold extremes expected in space. Electromagnetic interference (EMI) testing of the entire payload is done to ensure successful operation in the presence of EMI. Vibration testing is the third major environmental test, and this test ensures that the SDRs can withstand the vibration that occurs during launch. The SDRs are also tested with NASA's TDRS Satellites to ensure compatibility with the Space Network. Radiation tolerance is an important consideration for SDR operation in space. Typically, the individual parts are radiation tested before being put into an SDR. Parts are that are radiation tolerant or radiation hardened with well understood and tested behavior are procured for space SDRs. SDRs are not, at the box level, radiation tested.

\section{SDR Test Approach}

While the standard space radio test approach is sufficient if there is no intent to load a new waveform that operates beyond the bounds of the initial waveform, it may not be sufficient for future waveforms that expand the capabilities of the SDR.

A radio's performance will vary as a function of temperature if not properly compensated, be it with hardware or software. In the case of an SDR, this variance may affect a future waveform's performance, but without characterization in a thermal vacuum environment, the effects will be unknown. A reference oscillator's output frequency will vary depending on the temperature. The signal delay through the FPGA is dependent on the routing (which is waveform dependent) and the temperature. [8] The output power and noise figure will also change based on temperature. The impact of these temperature-dependent items must be understood, or at least bounded, because it is unlikely that a duplicate flight system will be procured to repeat the thermal testing for each new waveform.

An SDR's electromagnetic compatibility is also waveform dependent. If the center frequency of a new waveform is different than the initial waveform, the SDR could be susceptible to EMI when operating with the new waveform. The new waveform could also cause the SDR to emit a spur at a new frequency.

A preferred test approach for a software defined radio is to perform tests that characterize the performance of the platform across its range of capabilities in addition to the typical waveform tests that are completed on the initial waveform. Platform characterization tests should be done independently of the initial waveform and allow for development of future waveforms the SDR is deployed.

Space qualifying and certifying a software defined radio for use with the NASA Space Network combines the processes for a hardware radio with software development approaches. [9] As previously mentioned, all NASA radios are tested with the Space Network prior to launch. However, updating the radio certifications for new waveforms is also required. 
Ground certification tests will be completed for each new waveform and the results will be documented to show that the new waveforms still fall under the scope of the original certification. These tests will be completed on the engineering models for the SDRs and will not be done in a thermal vacuum chamber. The SCaN Testbed processes the firmware for the software defined radio using the NASA software development standards but adjustments were needed for the unique SDR firmware aspects of the process.

\section{SCaN Testbed Test Approach}

The testing of the three SDRs for the SCaN Testbed ranged from detailed platform characterization for the JPL SDR to very limited platform characterization for the GD SDR.

The GD SDR was the $5^{\text {th }}$ generation specified to work with the TDRSS S-band system. Test plans, procedures, and overall approach for the earlier generation hardware-based transceivers was used by the vendor and by NASA. Because this SDR had an extended list of operational modes, some insight into how the SDR would perform across a range of capabilities could be obtained.

\section{General DYNAMICS SDR TeSt SUMMARY}

The GD SDR testing at GRC focused on testing the prelaunch waveforms that were delivered with the GD SDR. The waveforms are compatible with TDRSS and contain many different reconfigurable parameters. Since there was no platform test waveform, it was difficult to test platform functions independently of the TDRSS waveform. The next three sections highlight some of the significant GD SDR testing, as well as recommendations for changes to future SDR testing.

\section{GD SDR AGC Characterization Testing}

The GD SDR analog and digital automatic gain controls (AGCs) were characterized [10] for all eight receive waveforms over temperature ranges from $-20{ }^{\circ} \mathrm{C}$ to $+60{ }^{\circ} \mathrm{C}$ and SDR input power levels from $-130 \mathrm{dBm}$ (the operational floor of the GD SDR) to $-90 \mathrm{dBm}$ (the highest expected operational receive power level). The tests were done in both an open room at ambient temperatures as well as in a thermal vacuum chamber where then temperature could be controlled to reach the hot and cold extreme temperatures expected in space $\left(-20{ }^{\circ} \mathrm{C}\right.$ to $\left.+60{ }^{\circ} \mathrm{C}\right)$. This characterization testing was used to create several algorithms to estimate the SDR received power, which is a desirable capability for on orbit characterization. The AGC response to varying SDR input power was different for all eight receive waveforms.

An SDR typically can have many more reconfigurable parameters than a fixed hardware transceiver, and the GD SDR is a prime example of that. The numerous reconfigurable modes can very quickly increase the amount of pre-flight verification testing that must be completed.
While the SDR receive power was characterized for the eight launch waveforms, it will be difficult to estimate the SDR input power for new receive waveforms that have not been characterized on the ground. A recommendation to solve both of these problems would be to create a platform independent waveform that would measure SDR input power and AGC characterization testing that is independent of the waveform configuration. This idea can be expanded to include all parameters that can vary with the waveform configuration and the temperature, such as the transponder coherent turn-around time. This would greatly decrease the amount of testing to be completed and prepare for new waveforms to be uploaded post launch.

\section{GD SDR Reconfigurable Parameters}

The GD SDR has the most unique combinations of receive and transmit waveforms out of all three SCaN Testbed SDRs. Each parameter can be changed individually and this can lead to many different operational states of the SDR. The large number of reconfigurable parameters led to a very large number of pre-launch verification and characterization tests. The testing completed at GRC was done from a black box perspective - very little knowledge of the design implementation was known. In order to reduce the number of tests, assumptions about the design were made. For example, the GD SDR receive waveform has two user data options. It can synchronize to a pseudorandom bit sequence and calculate the BER or it can pass framed data on to the Avionics for processing. It was assumed that the BER would be the same in both cases and so BER curves were taken in the first mode for user data. It turned out that the GD SDR did not process the bits in the same manner for both user data modes and the BERs were different.

The lesson learned here is that testing must be completed in all operational modes of the SDR in order to verify correct operation. Configurations that are not tested have a higher risk of problems, so reconfigurable parameters should be limited by the amount of test time available and the level of risk assumed. Finally, the testing matrix should be created with design knowledge of the SDR waveform in order to better down select the waveform configurations to be tested.

\section{Repairs Enabled by Software/Firmware Upgrades}

One of the benefits of a SDR is the ability to load new software post-launch. During the pre-launch verification and characterization testing phases at both GRC and GD, several software updates occurred in order to fix problems that were detected. In total, four software updates occurred on the GD SDR between July 2010 and August 2011. The updates fixed the following problems:

(1) The error detection and correction (EDAC) algorithm located in the GD SDR OE was initially reading the wrong location in memory and displayed both uncorrectable and correctable EDACs upon startup.

(2) The receive waveform operated differently when operating in simplex than in full duplex. It was able to 
maintain acquisition to lower SDR input power levels when operating in simplex.

(3) When operating in a non-coherent TDRSS mode [2], changes in acquisition of the receive waveform caused a reloading of the transmitter baseband filters.

(4) The framed user data interface over SpaceWire to the Avionics was initially not functional in both the transmit and receive waveforms.

(5) The BER curves were different when operating the receive waveform with the data sink set to SDR internal and with the data sent to the Avionics.

\section{JPL SDR TEST SUMMARY}

\section{Background}

The JPL SDR consists of hardware and software that was developed by multiple entities. The platform, which consists of the hardware and Operating Environment (OE), was procured through a NASA contract with the Jet Propulsion Laboratory (JPL). JPL designed and built the Baseband Processor Module (BPM), which includes the FPGAs and general purpose processor (GPP), and the GPS receiver module (GPSM), which includes the L-Band RF hardware. JPL contracted with L3/Cincinnati Electronics (L3/CE) to develop the S-band RF hardware. L3 also performed final hardware integration and box level environmental tests. In parallel, JPL developed the OE. The OE was integrated onto the platform after $\mathrm{CE}$ completed the hardware integration and test.

The SDR launched to ISS in July 2012 consists of the above hardware, the operating environment, 2 test waveforms, and 1 space network (SN) and near earth network (NEN) waveform. The SN/NEN waveform is compatible with NASA's tracking and data relay satellite system (TDRSS). The S-band test waveform performs sampling and playback for S-band, while the L-band test waveform performs only sampling for L-band. The SN/NEN-compatible waveform, the Glenn/Goddard TDRSS (GGT) waveform, was developed by the Goddard Space Flight Center and Glenn Research Center. GRC was responsible for the final integration of the waveform with the platform and all system level testing.

\section{Characterization Approach}

Having multiple waveform applications available increased the complexity of ground characterization. The GGT waveform was tested primarily with a TDRSS simulator, which produces and receives signals in a similar manner to a satellite and ground modems in the Space Network. The test waveforms were tested primarily with vector signal generator sources, which produce high-fidelity input tones that can be observed using the recording/capture waveform function. Test waveform playback, for S-band, was used sparingly since most focus was given to the operational GGT transmit waveform application capability.

The amount of time spent testing each waveform does not necessarily represent the usefulness of the waveform. The system level testing focused on addressing TDRSS compatibility questions - frequency stability, transmit power, spurious signal emission/susceptibility, etc. Since the GGT waveform is the operational launch waveform, most of the time was spent characterizing its performance. However, the signal processing of GGT makes it difficult to later determine underlying platform characteristics, which often would have been better understood through test waveforms.

\section{Applicability to Checkout and Commissioning}

The SCaN Testbed underwent several months of checkout and commissioning activities prior to beginning experiments. In the radio frequency world, these tests traditionally verify that radio and payload characteristics are similar to ground performance, including noise figure, emitted isotropic radiated power (EIRP), etc. A number of ground tests have proven useful for these purposes.

On the receive side, platform power and frequency characterization is critical. Power characterization should allow an operator to determine the platform received power from some telemetry or post-processed data. Namely, this includes understanding the platform gain control variation over temperature as well as the expected intermediate frequency (IF) spectrum power and the signal received at the analog-to-digital converter (ADC). For the JPL SDR, the S-band test waveform captures raw samples from the ADC, which provides a means of observing voltage and spectrum received on-orbit. The translation of a received power through the gain control and into a carrier-to-noise-density value in the ADC spectrum is critical to performing received power analysis. Additionally, characterization of the platform oscillator over time and temperature is useful when observing received frequency. The Doppler offset provides an indicator of whether the platform oscillator has drifted (when satellite-based compensation is enabled), and it can provide an indication of when the measurement was taken (without satellite-based compensation) due to the predicted relative velocity of the platform.

On the transmit side, the power amplifier (PA) output power and compression curve are two useful metrics. The S-band test waveform allowed the PA to be driven with a sine wave of arbitrary power for characterization purposes. Using this approach, as well as careful estimation of the drive power based on the voltage generated by the digital-to-analog converter (DAC), compression curves were developed to allow optimal tuning of the PA for different modulation schemes. The platform includes a transmitted power sensor, which essentially measures voltage on the PA output. The GGT waveform uses this sensor in a feedback loop, allowing the waveform to tune its power to the desired operating point of the PA. The sensor also allows ground 
operators to predict on-orbit transmitted power, which can be confirmed by the receiving satellite or ground station.

The GGT waveform exercises both receive and transmit characteristics, including data transfer between the radio and avionics. This makes it less useful for checkout of platform characteristics on-orbit, since much of the underlying platform behavior is abstracted away (through automatic gain control and transmit power feedback, for example). However, GGT performs pseudo-random binary sequence (PRBS) tests that allow overall bit error rate (BER) characterization. The data source/sink can be internal or processed externally on the avionics computer. This data path checkout is not possible with the launched test waveforms.

\section{Applicability to Experiments}

Future experiments will have different RF and processing requirements than the waveforms that launched with the radio. It is not possible to predict ahead of time exactly what platform capability is needed (outside of a broad set of platform requirements), so the project has two approaches to the problem. The first approach is to characterize a basic set of platform capabilities on the flight model (FM) radio prior to launch. The second approach is to maintain an engineering model (EM) of the radio on the ground, while performing platform characterizations that allow a performance delta to be predicted.

A majority of the testing performed at GRC on the FM SDR characterized performance of the GGT waveform. These tests included acquisition frequency range, acquisition time, acquisition over frequency offset, BER performance on forward and return links, Doppler tracking rate and range, error vector magnitude (EVM), output frequency stability, receive performance in the presence of continuous wave (CW) and modulated interferers, loss of signal lock level, and transmitted spectrum and spectral mask compliance. Most of these tests exercise waveform-specific tracking loops and signal processing techniques. These tests form a benchmark from which future waveform enhancements can be compared.

Some tests were completed using the test waveforms or the GGT waveform in a non-standard configuration, using STRS ground or run tests. These tests include receiver noise figure and gain, vector modulator temperature compensation, oscillator temperature compensation, conversion of the transmit power sensor to engineering units over temperature, PA compression curves, and gain flatness. The temperature-dependent tests are important because they are specific to the sensor included on the FM radio; testing on the EM will likely not match the FM perfectly. Similarly, the noise figure, PA compression, and transmit power sensor tests allow a waveform designer insight into the expected on-orbit performance as well as key output target levels. The gain flatness test was performed across the full transmit spectrum, 2200 to $2300 \mathrm{MHz}$, and is useful for understanding what frequency-dependent transmit power compensation must be done for waveforms operating over a larger bandwidth or at a different center frequency (the hardware maximum intermediate frequency bandwidth is approximately $10 \mathrm{MHz}$ ).

Since there was only limited time available for radio characterization, the testing approach placed higher priority on the GGT waveform than on fully characterizing the platform. This was driven mainly by system-level testing (electromagnetic interference, TDRSS compatibility, thermal-vacuum) that did not distinguish between the platform and waveform. A large number of platform acceptance tests were performed at $\mathrm{L} 3 / \mathrm{CE}$, but these tests were focused on showing compliance with platform requirements by transmitting into a worst-case voltage standing wave ratio (VSWR) or receiving at the edges of the spectrum pass-band. As such, it is important that the end user of the SDR perform enough platform characterization to cover any expected future waveform development.

\section{Software Updates and Development}

The JPL SDR was launched with 4 distinct software components: the operating environment, the 2 tests waveforms, and the GGT waveform. The OE and test waveforms were not modified during any of the performance testing. The GGT waveform was modified multiple times to increase performance, support systemlevel integration activities, and improve hardware protection.

GGT performance improvements affected waveform tuning values, such as calibration tables and gain control targets. The temperature compensation table, which provides modulator scale factors and frequency offsets, was updated several times to reflect improved analysis throughout testing. The gain control target was updated for various waveform modes of operation (which encompass a mixture of data rate, modulation, and coding settings) to improve lock performance by increasing or reducing power levels at the ADC. An output level control feedback loop was incorporated, allowing the waveform to target a preestablished transmit power level.

Several waveform capabilities were added to address system-level integration activities. For example, bit error rate testing was improved by displaying a warning whenever the BER tester dropped lock. During EMI testing, a need was identified to report near-instantaneous bit errors exceeding a specific threshold, which would allow correlation of bit errors with electromagnetic interference at specific frequencies. During TDRSS compatibility testing, CW transmissions were used to establish carrier-to-noise ratio; this was improved by adding a waveform command that would transmit $\mathrm{CW}$ on the fly. A number of system tests were performed to improve the flow of SpaceWire data communication between the radio and the avionics processor; waveform enhancements were made to display extra debug information and overflow/underflow flags, which helped hardware driver improvement efforts. 
Hardware protection was enhanced throughout characterization testing. The platform sensor values were processed through an averaging routine to reduce observed noise and improve monitoring efforts. The PA activation was restricted by the waveform to prevent the PA from operating if the drive level was beyond vendor's limits. A FPGA register check was added to allow ground operators to verify the FPGA was set correctly for the loaded mode; this could also be used to evaluate whether a single event upset (SEU) has occurred on orbit.

\section{HARRIS SDR TEST SUMMARY}

\section{Test Discussion}

The Harris SDR was delivered with an integrated, TDRSScompatible waveform, and was tested by the vendor using similar tests for a hardware-based transceiver. The waveform is flexible and has many reconfigurable parameters, such as variable data rates, forward error correction, framing, and randomization options. The waveform exercises a subset of the platform's capabilities. For example, the platform's transmit and receive center frequencies are tunable over $500 \mathrm{MHz}$ within the Space Network allocation; however, the waveform only operates with a single transmit/receive frequency pair. Adding support for additional operational frequencies would better characterize the platform, at the expense of a larger test matrix. The aggressive schedule for the development and test of the Harris SDR did not allow for these additional modes of operation. Instead, if a simpler platform characterization waveform was used, this characterization over frequency would be more practical. This could be implemented as a separate test waveform application, or a STRS RunTest within the existing waveform application.

Another consideration during testing was the intended use of higher-order modulation schemes for future experiments. This was considered early in the flight system integration process, to ensure that the system would support higherorder modulations. A simulation model of the transmit system was developed to evaluate the predicted performance, as well as a thermal model to account for temperature variations. The model will help determine how to dynamically adjust the SDR's output power over temperature to maintain operation in the amplifier's linear region. Determining the accuracy of the model will be left to future experiments. A platform characterization test which varies the Harris SDR's drive level to the TWTA during the Thermal Vacuum testing would have been the ideal method to measure output power variation and to refine the model predictions.

Furthermore, the phase and gain response of the transmit system using the launched waveform were outside the Space Network specification. An analysis was performed by the Space Network team to evaluate impact on performance and it was determined that there was minor impact to performance with the current waveform. Determining the impact on higher-order modulation schemes will require further analysis.

The Harris SDR contains a Digital Signal Processor (DSP) on the modem processing board. The waveform that was developed for the launched configuration does not use the DSP. Harris Corporation ran a test script during environmental testing to exercise the DSP, but NASA did not perform any additional DSP testing during the system level environmental tests, with the exception of the SDR's built-in-test capabilities. The recommendation is to further test all the platform's resources throughout the system level integration.

Limited testing across the full transmit and receive frequency bands was performed on the Harris SDR and the associated RF subsystem. S-parameters of components and systems were measured across the full modulation bandwidth, but testing was restricted to the baseline center frequencies. For example, the noise figure of the receiver low noise amplifier (LNA), and the amplitude modulation (AM) to phase modulation (PM) conversion of the transmitter were characterized over the full bandwidth, but with the radio tuned to a single center frequency. Performance across the entire tunable frequency band can only be estimated from the available data. The Ka-band TWTA was characterized at three distinct frequencies, one of which is the baseline center frequency. Determining the performance across the full range of potential frequencies can no longer be easily measured and will require further analysis.

\section{Repairs Enabled by Software/Firmware Upgrades}

\section{1) Double NRZ-M}

During compatibility testing with the Space Network, it was discovered that the NRZ-L/M encoding algorithm, implemented in an FPGA in the Harris SDR, was not implemented as designed. When the SDR was programmed to be NRZ-M, it converted both the serial data from NRZ-L to NRZ-M and then converted to NRZ-M again in each I/Q leg of the modulator. This firmware error was not noticed during local testing performed prior to the compatibility testing, as the local test equipment was configured to decode back from NRZ-M in the same order. The firmware was modified, tested, and delivered from Harris, verified in the GRC Ground Integration Unit, and uploaded to the flight system within a month of discovering the error. If this were a fixed hardware implementation, unique Space Network configurations at WSC would have been needed to accommodate the error and performance would have suffered.

\section{2) BER flaring}

When performing numerous Bit Error Rate (BER) tests as a function of signal-to-noise ratio (SNR), it was discovered that occasionally the BER of the system at the higher SNRs was much worse than expected. This seemed to be a 
random occurrence. Harris was consulted, and it was discovered that there was not enough margin in the timing sequences between initiating the parameters for the Analog to Digital Converter (ADC) and the parameters for the FPGA. A delay parameter was identified as the value to be set to control the timing between the two components, but the setting of the delay value required optimization and is dependent on the voltage and temperature of the system. Adjusting the delay and re-running the BER curve would have been very time consuming and the results would be skewed by the randomness of the event. Instead, the components of interest were modeled and the ADC vendor was consulted to validate the model. Various delay values were tested on the flight model of the Harris SDR to confirm the behavior predicted by the model. An ideal delay value was selected, the firmware was updated, and the unexpected BER performance has not reoccurred.

\section{3) Watchdog reboot issue}

The Harris SDR unexpectedly rebooted several times during routine testing. The cause was traced to a race condition in the commanding sequences. Whenever a command is sent by Avionics, a response is sent back by the SDR. When that response is received, the next message is sent. At the point where the last packet from response 1 is complete and the start of message 2 is sent, the SDR takes a small amount of time to be ready for the next message. Adjustments were made in the timing algorithm implemented in the SDR software and the problem has been corrected.

\section{SUMMARY AND CONCLUSION}

This paper describes the approach used for the testing and characterization of the three SDRs on the SCAN Testbed. It describes tests that would be typically performed on a hardware radio and also proposes additional platform tests that should be done to characterize an SDR to enable future waveforms to be developed after deployment. The experience performing platform-only and integrated platform testing for the $\mathrm{SCaN}$ Testbed highlights the need for the development of a test waveform to be used to characterize the SDR system independent of a communication waveform. The capability of the test waveform to perform both transmit and receive tests across the range of the platform capabilities is suggested in the paper. It also highlights some tests that were completed for each of the three SCaN Testbed SDRs and describes the updates that were enabled by utilizing the reprogramming capability of an SDR.

\section{FUTURE WORK}

At the completion of the checkout and commissioning activities, which have the primary objective of assuring that the system operating on the ISS performs as predicted by ground testing, a suite of tests will be conducted to fully characterize the on-orbit performance of the system over time. Tests described in this paper that can be conducted in the operational environment will be repeated using the onorbit system. These will include BER curves using the dynamic range provide by the change in path length, antenna gain, and possibly mispointing the tracking antenna to provide additional loss. ADC captures will be obtained and converted to spectrums using FFTs implemented either on-orbit and on the ground to understand the interference environment. Transmit spectrums will be obtained on the ground. New waveforms will be installed on the flight SDRs and new lessons on implementing these will be captured and published.

\section{REFERENCES}

[1] SCaN Testbed Experiment and System Information: http://spaceflightsystems.grc.nasa.gov/SOPO/SCO/SCaNTest bed/Candidate/

[2] Space Network (SN) User's Guide, 450-SNUG (Revision 9 , August 2007).

[3] R. Reinhart, T. Kacpura, L. Handler, C. Hall, D. Mortensen, S. Johnson, et.al. Space Telecommunications Radio System (STRS) Architecture Standard, Release 1.02.1. NASA Technical Memorandum 2010-216809, December 2010.

[4] Electra radio: http://mars.jpl.nasa.gov/mro/mission/instruments/electra/

[5] "Space Software Defined Radio Characterization to Enable Reuse, Dale Mortensen, Dan Bishop, Vantage Partners LLC, David Chelmins, NASA GRC - USA, 30th AIAA International Communications Satellite System Conference, September 24-27, 2012, Ottawa, Canada.

[6] Pre-flight Testing and Performance of a Ka-band Software Defined Radio, Joseph Downey, Richard Reinhart, Thomas Kacpura, NASA GRC - USA, 30th AIAA International Communications Satellite System Conference, September 24-27, 2012, Ottawa, Canada.

[7] "CoNNeCT's Approach for the Development of Three Software Defined Radios for Space Application", Sandra Johnson, Richard Reinhart, Thomas Kacpura, NASA GRC USA, 2012 IEEE Aerospace Conference, March 3-10, 2012, Big Sky, Montana.

[8] "Direct Measures of Path Delays on Commercial FPGA Chips", Mattia Ruffoni, Alessandro Bogliolo, Italy (spi02b.pdf)

[9] Difficulties in Providing Certification and Assurance for Software Defined Radios, John Giacomoni, Douglas C. 
Sicker, University of Colorado at Boulder, IEEE 1-4244-0139.

[10] GD SDR Automatic Gain Control Characterization Testing." , to be presented at the Wireless Innovation Conference in Washington, D. C., January 2013.

\section{Biographies}

David Chelmins received a B.S. in Computer Engineering from Ohio Northern University in 2008 and a M.S. in Electrical and Computer Engineering from the Georgia Institute of Technology in 2012. He is a research computer engineer with the Digital Communications and Navigation branch at the NASA Glenn Research Center. Currently, David is the project experiments lead and JPL SDR subject matter expert for the Space Communications and Navigation (SCaN) Testbed project.

Joseph Downey received a B.S. in Electrical Engineering from the University of Toledo in 2008 and is currently working towards a M.S. in Electrical and Computer Engineering from the Georgia Institute of Technology. Since 2008 he has worked at the NASA Glenn Research Center in the field of space-based software-defined radio technology. His current work includes efficient FPGA implementations of high-rate spectral-efficient communication techniques.

Sandra K. Johnson received a BSEE from The Ohio State University in 1988 and a MSEE degree from University of Akron in 2000. She has worked with space communications technology since 1990. Sandra was a coprincipal investigator for the Space Telecommunications Radio Standard (STRS) development and served as cochair of the Wireless Innovation (SDR) Forum Space Work Group from 2004-2007. Currently, Ms. Johnson is the deputy principal investigator for the SCaN Testbed Mission.

Jennifer M. Nappier received a B.S. in Electrical and Computer Engineering from Ohio State University in 2006 and a M.S. in System and Controls Engineering from Case Western Reserve University in 2009. She has worked for NASA Glenn Research Center since 2005 and is currently the GD SDR S-Band experiment principle investigator for the SCaN Testbed. 